\title{
Mapping Facial Expression to Internal States Based on Intuitive Parenting
}

\author{
Ayako Watanabe, Masaki Ogino, Minoru Asada,
}

\begin{abstract}
Sympathy is a key issue in interaction and communication between robots and their users. In developmental psychology, intuitive parenting is considered the maternal scaffolding upon which children develop sympathy when caregivers mimick or exaggerate the child's emotional facial expressions [1]. We model human intuitive parenting using a robot that associates a caregiver's mimicked or exaggerated facial expressions with the robot's internal state to learn a sympathetic response. The internal state space and facial expressions are defined using psychological studies and change dynamically in response to external stimuli. After learning, the robot responds to the caregiver's internal state by observing human facial expressions. The robot then expresses its own internal state facially if synchronization evokes a response to the caregiver's internal state.
\end{abstract}

\section{INTRODUCTION}

Sympathy is indispensable for communication. Although it is unclear how sympathetic feelings are evoked, facial expressions are an important cue for eliciting sympathy. Of the communication channels used by human begings, $55 \%$ are related to the face, $38 \%$ to the tone of voice, and $7 \%$ to verbal information [2]. It is not clear how the capacity for sympathy based on facial expression is formed. Infants instinctively respond to faces, and babies a few days old distinguish their mother's face from those of others after being contact with their parent for 11 or 12 hours [3]. Conversely, an investigation of newborn facial expressions showed that the basic facial expressions are innate [4]. To realize natural communication between robots and their users, the processes underlying how these essential abilities are combined to elicit sympathy must be clarified.

Human-like robots able to show distinct facial expressions have been developed [5] [6], but facial expressions to be used in specific situations are specified explicitly by the designer in advance, leaving robots unable to adapt to nonspecified situations, and unable to modify their internal state in response to the facial expressions of users. Breazeal et al. proposed a developmental model that enables a robot to derive the relationship between motor commands for its facial expressions

Ayako Watanabe is with Graduate School of Engineering, OsakaUniversity, 2-1 Yamadaoka, Suita Osaka, Japan (corresponding author to provide phone: +81-6-6879-7349; fax: +81-6-6879-7348; e-mail: watanabe@er.ams.eng.osaka-u.ac.jp)

Masaki Ogino is with Asada Synergistic Intelligence Project, ERATO, Japan Science and Technology Agency, FRC1, Graduate School of Engineering, Osaka-Univ. 2-1 Yamadaoka, Suita Osaka, Japan (corresponding author to provide phone: +81-6-6876-8884; fax: +81-6-6876-8994; e-mail: ogino@jeap.org)

Minoru Asada is with Asada Synergistic Intelligence Project, ERATO, Japan Science and Technology Agency, FRC1, Graduate School of Engineering, Osaka-Univ. 2-1 Yamadaoka, Suita Osaka, Japan. He is also with Osaka University, 2-1 Yamadaoka, Suita Osaka, Japan (e-mail:asada@jeap.org) and those of the caregiver's by imitating facial expressions during the robot's motor babbling [7]. Sympathy, however, does not involve simply mimicking the facial expressions of others. More important is the ability to evoke the same internal state as others based on the facial expressions and vocal characteristics of others. Kobayashi et al. [8] proposed learning in which the robot categorizes a user's facial expressions under given emotional labels. This enables a robot to evoke the same emotional label felt when the caregiver has touched the robot before. Again, however, emotional labels are fixed and the caregiver's active synchronization is not considered.

How do human children develop sympathy through interactions with their caregivers? In developmental psychology, the caregiver behavior called "intuitive parenting" [9] serves as a "maternal scaffolding" upon which children develop sympathy as they grow. A typical example is when caregivers mimic or exaggerate a child's emotional expressions. This is considered a good opportunity for teaching children how to feel in realtime [10], and most adults possess this skill. Children are thus able to understand the meaning of facial expressions and develop sympathy toward others as the process is reinforced through emphasis on the facial expressions of their caregivers. This is because children empirically learn the connection between their internal state and the facial expressions of others.

We considered applying intuitive parenting to user-robot interactions to realize sympathetic behavior by robots within the context of cognitive developmental robotics [11]. Two design issues arise in cognitive developmental robotics: one is the structure of the embedded behaviors in the robot brain such as learning, and the other is the issue of environmental design, including human behavior toward robots. Intuitive parenting is considered typical of the issue of environmental design, which is used to modify the robot's internal structure to elicit sympathetic behavior toward others.

To realize this, we propose a communication model that enables a robot to associate facial expressions with internal states through intuitive parenting by users who mimic or exaggerate a robot's facial expression. The robot strengthens the connection between its internal state and the facial expression associated with a particular state. The internal state of a robot and its facial expressions change dynamically depending on the external stimuli. After learning, facial expressions and internal states are classified and made to mutually correspond by strengthened connections. When a user shows a facial expression to the robot, the robot evokes a human internal state based on the human facial expression, which then elicits a sympathetic expression because the robot's internal state is 
indirectly affected by the human internal state.

\section{LEARNING THROUGH INTUITIVE PARENTING}

\section{A. Intuitive parenting}

"Intuitive parenting" is a typical attitude adopted by a caregiver toward a child. The caregiver may, for example, mimic or exaggerate facial expressions of the child to sympathize with the child's internal state when the child is experiencing a particular emotion. Through such experiences, the child is expected to learn the association between the condition experienced and the caregiver's facial expression at that time.



Fig. 1. Learning model for developing sympathy in children through intuitive parenting

Fig. 1 shows a learning model for a child developing a sense of sympathy through the intuitive parenting of its caregiver. When a child undergoes an emotional experience and expresses its feelings by changing its facial expression, a caregiver sympathizes with the child and shows the child a concomitantly exaggerated facial expression. The child then discovers the relationship between the emotion experienced and the caregiver's facial expression and comes to mutually associate the emotion and the facial expression. The emotion space in this figure is constructed based on the model proposed by Russell [12].

Stimulus sharing, one of the sources of sympathy, is categorized as either incidental or intentional in intuitive parenting. A typical example of incidental case is a sudden sensation when exposed to a modality such as a loud noise or a flash of light. The child and caregiver are surprised simultaneously, and as the caregiver talks to the child, the surprise is evident in their facial expressions. A typical example of the intentional case is when the caregiver shows the child an entertaining and smiles to stimulate the child's interest at the same time.

\section{B. Identifying problems}

We propose several assumptions about the robot and caregiver that are required for implementing the model.

a) Robot: When the internal state of a robot changes in response to an external stimulus, the robot's facial expression changes correspondingly. The robot is also able to observe the caregiver's facial expressions and has the ability to associate its internal state with the facial expression of the caregiver.

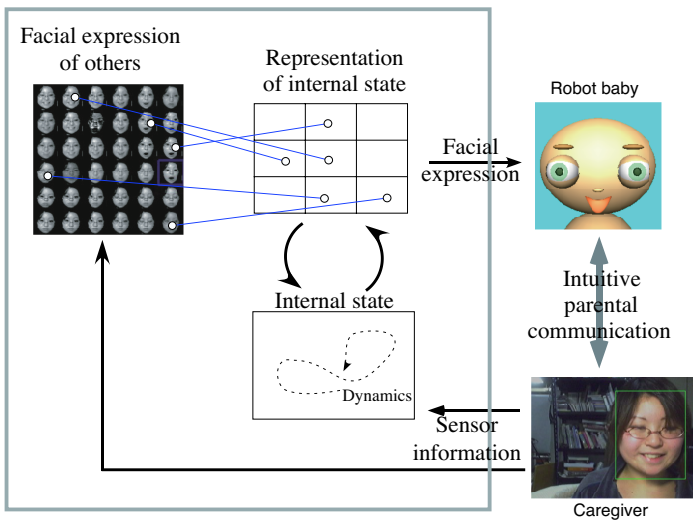

Fig. 2. Associating visual facial expressions of others with internal states

b) Caregiver: The robot's human caregiver infers changes in the robot's internal state based on shared experiences and the robot's facial expression. The caregiver responds to the robot with intuitive parenting. The caregiver observes the robot's facial expression or condition, then responds with a corresponding facial expression.

\section{System overview}

Our proposed virtual robot (Fig. 2) acquires sensory information from the caregiver, including touch sensors (keyboards), sounds, and camera images. Changes in sensor information change the robot's internal state, which consists of two independent variables, the arousal-sleep axis and the pleasuredispleasure axis [12]. These internal variables change based on simple relaxation dynamics equations. This internal state is represented in two-dimensional space associated with the representational space of others' facial expressions through intuitive parenting communication as follows:

1) When the caregiver touches sensors or makes a noise, the robot's internal state changes.

2) The robot shows a facial expression based on its internal state. The association between the internal state and this facial expression is based on Yamada's model [13] (Fig. 5).

3) The caregiver imitates the robot's facial expression. The robot detects the change in the caregiver's facial expression and its internal state in representational space, and associates these changes with each other.

\section{Internal state}

The robot's internal state is constructed based on the human affect model. Of the many numerous human affect models proposed, we use the circumplex model of the affect proposed by Russell [12] because correspondence between the internal state and facial expressions is relatively simple as explained later, and we add the dynamics property to Russell's affect model.

Russell's model assumes that the "arousal-sleep" axis and "pleasure-displeasure" axis are the most basic dimensions and that numerous different affect categories are assigned to each (Fig. 3). 


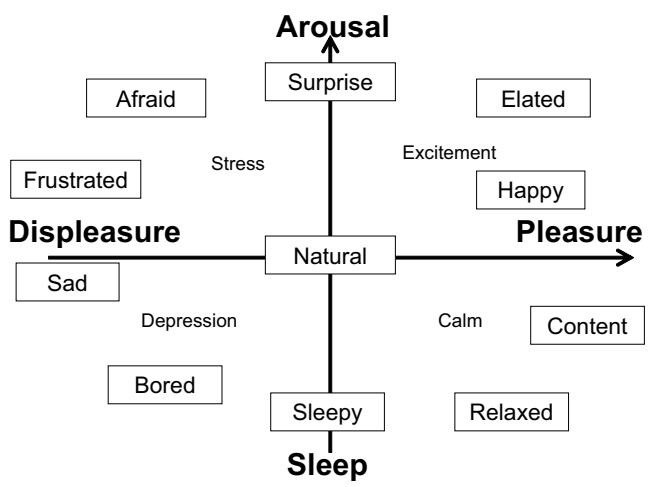

Fig. 3. Circumplex model of affect [Russell 1980]

The robot's internal state is modeled using this twodimensional affect model. Because few opportunities arise to learn the sleepy facial expressions of others through intuitive parenting, the sleep axis is omitted here. The robot's internal space (Fig. 4) is sequential and represented using twodimensional space.

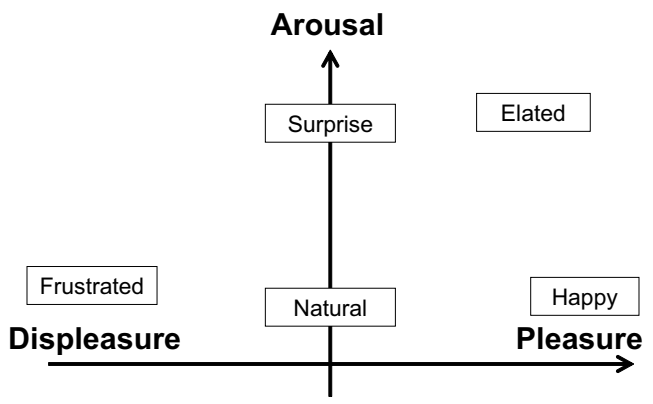

Fig. 4. Internal space of robot

The robot's internal state $S$, consisting of two independent variables is expressed as follows:

$$
\boldsymbol{S}=(p, a) \quad\left\{\begin{array}{c}
(-1<p<1) \\
(0<a<1)
\end{array},\right.
$$

where $p$ indicates the level of pleasure and $a$ the level of arousal located in space represented in Fig. 4. The robot expresses its internal state in its facial expression, and learns the connection between the internal state and the human facial expression.

The robot's internal state changes as a relaxation dynamics equation:

$$
\begin{aligned}
\boldsymbol{\tau} \dot{\boldsymbol{S}} & =-\boldsymbol{S}+\Sigma \boldsymbol{r}_{i}+\boldsymbol{S}_{0} \\
\Sigma \boldsymbol{r}_{i} & =\boldsymbol{r}_{e}+\boldsymbol{r}_{o}
\end{aligned}
$$

where $\boldsymbol{S}_{0}$ is the internal state for the original point, $\tau$ the decay time constant, $\boldsymbol{r}_{e}$ the effect of the external stimulus, and $\boldsymbol{r}_{o}$ the effect induced by the caregiver's expression. When a caregiver provides the robot with a stimulus, $\boldsymbol{r}_{e}$ becomes a nonzero vector. The value of this vector changes based on stimulus categories and the duration over which the stimulus is given. $x$ is a stimulus category and $\phi_{x}$ is the duration. $\boldsymbol{r}_{x}$ is the constant vector decided by stimulus $x$.

$$
\boldsymbol{r}_{e}=\boldsymbol{r}_{x} e^{-A \phi_{x}}
$$

While the caregiver's facial expression and internal state are being learned and mapped, $\boldsymbol{r}_{o}$ is 0 . After learning, $\boldsymbol{r}_{o}$ takes values that depend on the learned association between the caregiver's facial expression and the robot's internal state.

\section{E. Facial expression}

Yamada et al. [13] proposed that a relationship exists between the relative displacement of facial expressions and basic affect categories, and that the relationship is expressed using two variables, "Curving and releasing" and "Inclination" (Fig. 5).

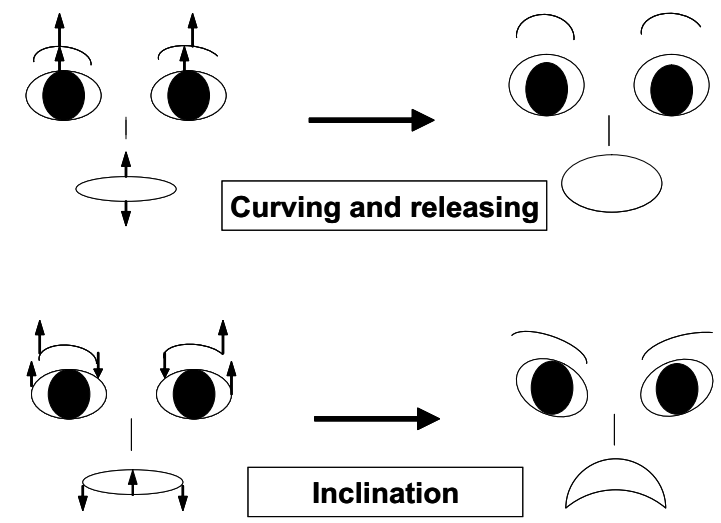

Fig. 5. Two structural variables for facial expression: "curving and releasing" and "inclination" [Yamada 1993]

Curving and releasing involves displacement of feature points related to the amount of eyebrow curving and eye and mouth opening. Inclination involves the displacement of the feature point concerned with the angles of the eyes and eyebrows, and the extent of the $\mathrm{V}$ or inverted $\mathrm{V}$-formation of the mouth. Feature point displacement is related to the level of arousal, and the extent of $\mathrm{V}$ formation of the mouth is related to the level of displeasure.

The robot changes its facial expression based on its internal state, $\boldsymbol{S}$. Despite a good correspondence with the requirements set out in Yamada's study, robotic facial expressions were unnatural, so we defined proportionality factor $b$ and modified it slightly in each quadrant. The parameters changing the robot's facial expression are shown below.

The robot displaced its eyebrows, eyelids, eyes, and mouth within a finite range.

$$
b= \begin{cases}0 & (0<p) \\ 1 & (0>p)\end{cases}
$$




$$
\begin{aligned}
\left(\begin{array}{l}
h_{\text {mouthdown }} \\
h_{\text {mouthside }} \\
h_{\text {browin }} \\
h_{\text {browout }} \\
h_{\text {eyeliddown }} \\
h_{\text {eyelidrotate }}
\end{array}\right) & =\left(\begin{array}{c}
1 \\
1 \\
0.5 b \\
-0.5 b \\
-0.4 b \\
-b
\end{array}\right) p+\left(\begin{array}{c}
1 \\
0 \\
1 \\
1 \\
-0.4 \\
0
\end{array}\right) a \\
& =\boldsymbol{U} p+\boldsymbol{V} a
\end{aligned}
$$

Fig. 6 shows the robot's facial expression when $S=(p, a)$ changes in relation to its internal state.

Eq.6 indicates that $\boldsymbol{U}$ is the facial expression vector related to curving and releasing and $\boldsymbol{V}$ thethe vector related to inclination. Fig. 7 shows the configuration of parameters for the robot's face and facial expressions for increasing curving and releasing or inclination.

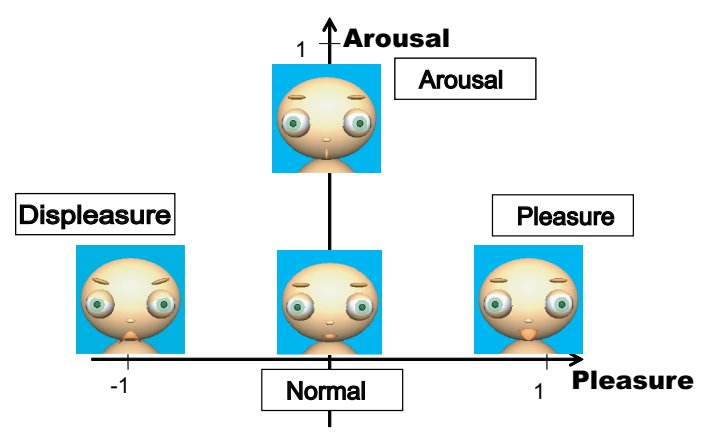

Fig. 6. Facial expressions and internal state

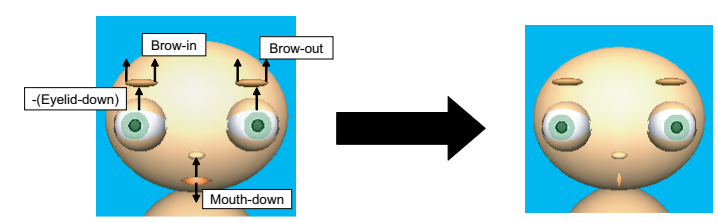

(a) Curving and releasing

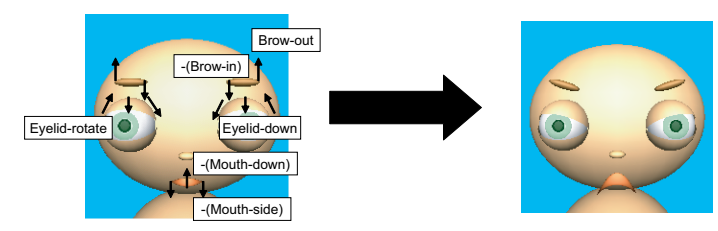

(b) Inclination

Fig. 7. Parameters for facial expressions

Using computer graphics, the robot's face was created to resemble a baby's face, because our learning model is based on child development interacting with caregivers in intuitive parenting. Infant faces have are characterized by a broad forehead and a large distance between the eyes [14].

\section{F. Facial expression recognition}

The face-colored area in the camera image is identified and normalized. Image luminance values are input to a self- organizing map (SOM) [15] to represent facial expressions of the caregiver. The SOM is taught in advance using input consisting of 500 images divided into five categories, each having images: normal, surprise, pleasure, displeasure, and laughter (Fig. 8). SOM images are classified based on basic

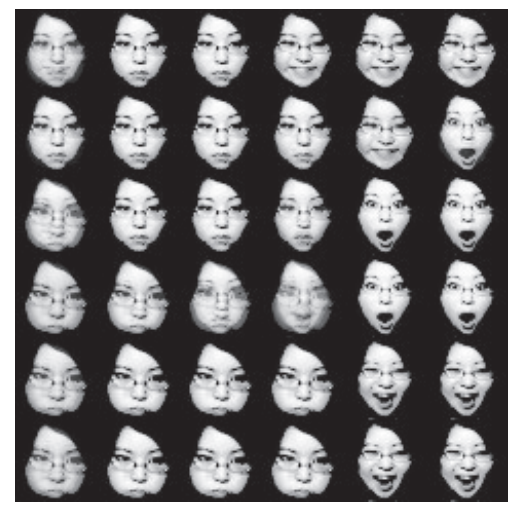

Fig. 8. Self-organizing map of caregiver's facial expressions

facial expressions. In Fig. 8, surprise faces are located at center right in the SOM, laugh faces at bottom right, displeasure faces at lower center, normal faces from upper left to the center, and pleasure faces at upper right.

When the robot began learning through interactions with a user, images of human facial expressions were input to the robot online. Video images are input from a camera in real time. The input image is extracted by color, the size is normalized, and the winner node on the SOM is determined. The winner node is calculated by searching for the vector that has the luminance value nearest to each of the SOM's constant vectors. The robot then matches that constant vector to the SOM's winner node.

\section{G. Association learning}

1) Representational space for internal state: Although the internal state is expressed using continuous variables, the internal state must be treated as a finite node number for Hebbian learning, so representational space for the internal state is prepared to facilitate Hebbian learning by the robot.

Fig. 9 shows an overview of Hebbian learning between the face SOM and internal space. Internal space is the representational space of the internal state. Connection weights are updated by evaluating the SOM node $f_{i j}(V)$ activity from an input image and node activity in representational space $g_{k l}(S)$ from the robot's internal state at that time.

This experiment assumes that the number of SOM nodes is the same as that of representational space in the internal state.

2) Hebbian learning: Internal state $\boldsymbol{S}$, is expressed in representational space as the activation level of discrete nodes $g_{k l}(\boldsymbol{S})$, determined as follows:

$$
g_{k l}(\boldsymbol{S})=e^{-\rho\left|\boldsymbol{S}-\boldsymbol{S}_{k l}^{r}\right|^{2}},
$$

where $\boldsymbol{S}_{k l}^{r}$ is the internal state attached to the $(k, l)$-th node in the representational space, represented as follows:

$$
\boldsymbol{S}_{k l}^{r}=\left(\begin{array}{c}
S_{k}^{r} \\
S_{l}^{r}
\end{array}\right)=\left(\begin{array}{c}
k / N_{P} \\
l / N_{A}
\end{array}\right)
$$




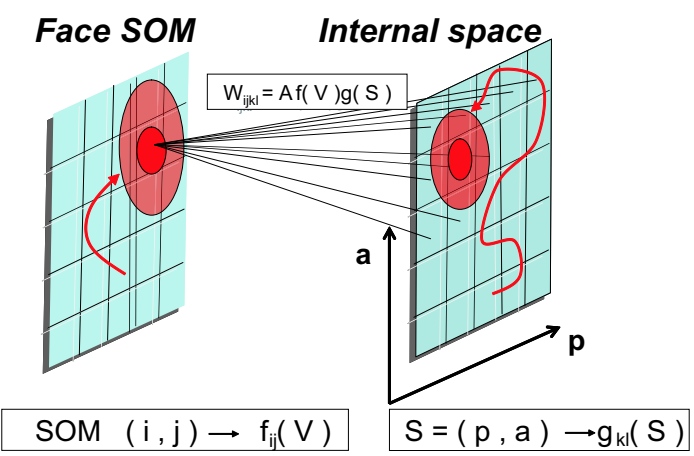

Fig. 9. Hebbian learning between the face SOM and internal space

$$
\left\{\begin{array}{c}
k:-N_{P} \cdots N_{P} \\
l: 0 \cdots \cdots N_{A}
\end{array}\right\}
$$

The caregiver's facial expression is represented as the activation level of the node in the SOM $f_{i j}(\boldsymbol{V})$. When facial expression $\boldsymbol{V}$ is input, the activation level is determined as follows:

$$
\begin{array}{lcc}
\delta_{i j} & = & \frac{1}{N} \sum_{k=0}^{N}\left|\boldsymbol{V}^{k}-\boldsymbol{V}_{i j}^{k}\right|, \\
f_{i j} & = & e^{-\nu \delta_{i j}^{2}} .
\end{array}
$$

where $\nu$ is a constant that describes an increase in SOM activity.

First, we calculated the difference in the luminance value between each constant vector and the input vector per pixel, i.e., the average of the difference between luminance information for the SOM and for input image $\delta_{i j}$. We then evaluated the node in the SOM that has the minimum mean difference as the winner node $\left(i_{\text {win }}, j_{\text {win }}\right)$ for an input image.

Connection weights between these maps, $w_{i j}^{k l}$, are updated by Hebbian learning as follows:

$$
\begin{aligned}
w_{i j}^{k l}(t+1) & =w_{i j}^{k l}(t)+\Delta w_{i j}^{k l} \\
\Delta w_{i j}^{k l} & =\alpha f_{i j}(V) g_{k l}(S),
\end{aligned}
$$

where $\alpha$ is the learning rate. $\alpha$ is attenuated as time goes by. $\lambda$ is a constant and $\mu$ is the attenuation rate over time.

$$
\alpha=\lambda e^{-\mu t} \quad(0<\lambda<1)
$$

After learning, $\boldsymbol{r}_{o}$ has several values that depend on the learned association between the caregiver's facial expression and the robot's internal state:

$$
\begin{gathered}
\left(k_{p}, l_{p}\right)=\underset{k, l}{\arg \max _{k, l}} w_{i j}^{k l} \\
\boldsymbol{r}_{o}=\beta \boldsymbol{S}_{k_{p} l_{p}}^{r}
\end{gathered}
$$

The winner node of the $\operatorname{SOM}(i, j)$ is calculated when a caregiver shows a facial expression to the robot. The node of the robot's internal state $\left(k_{p}, l_{p}\right)$ that has the highest weight $w_{i j}^{k l}$ associated with the winner node in the $\operatorname{SOM}(i, j)$ is then searched for. Representational vector $\boldsymbol{S}_{k_{p} l_{p}}^{r}$ attached to the winner node of the internal state $\left(k_{p}, l_{p}\right)$ effects the robot's internal state. The vector multiplied by $\beta$ is defined as variable vector $\boldsymbol{r}_{o}$ and is influenced by human facial expressions, which changes the robot's internal state.

\section{EXPERIMENTS}

\section{A. Experimental conditions}

In experiments, four categories of stimuli and five kinds of facial expressions are presented to the robot. The sequence in which different stimuli were presented was not fixed, but we did not apply the displeasure stimulus soon after the pleasure stimulus because this is considered unlikely in actual human interaction. Fig. 10 shows an example of exposure to a stimulus over time and the effect this has on the robot's internal state and the corresponding facial expression.

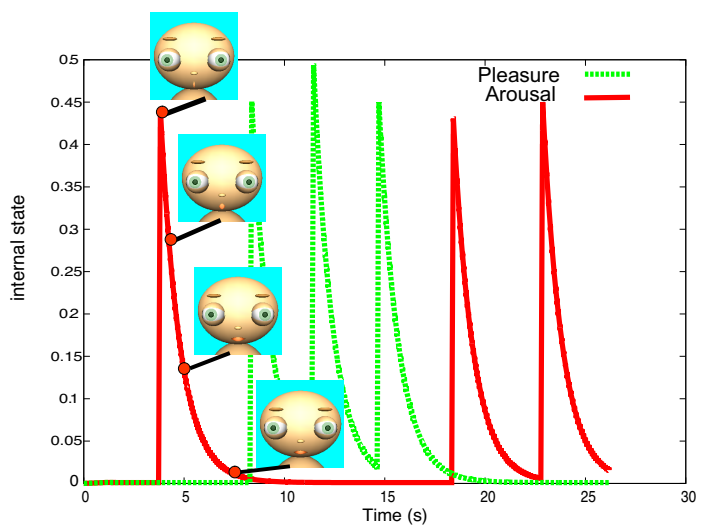

Fig. 10. Internal state

\section{B. Experimental conditions}

a) Robot: The robot we created using computer-graphics (OpenGL) consisted of a torso and moved its facial features within a finite range. It observed human facial expressions via a camera and sound with a microphone.

Input from a keyboard is used to simulate stimuli for the robot's touch sensors and is assumed the robot to distinguish between three different touch sensor inputs. Each touch sensor input effects the internal state differently, so pressing different keys inputs pressed different vector values into equation (3), yielding responses corresponding to pleasure, arousal, or displeasure.

b) Environment: A caregiver provides stimuli to the virtual robot using a computer. The caregiver also presents the robot with different facial expressions, which are then captured by a USB camera and used as input images for the robot (Fig. 11).

Fig. 12 shows an example of an input image captured by the camera. The green square indicates the face detected based on color luminescence.

Given that the experiment was conducted in an ordinary office environment, input information consisted of both the caregiver and other unknown environmental elements. A sound stimulus, for example, could be input in timing unintended by the person. Fig. 13 shows the transition in the internal state during learning. Yellow arrows indicate the application of external stimuli by the caregiver or circumstance. 


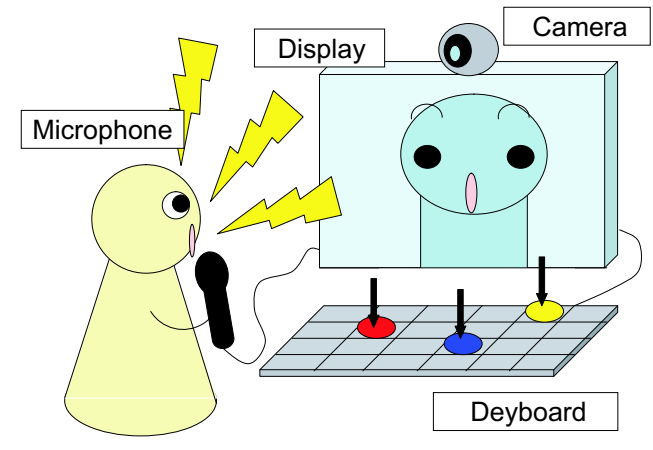

Fig. 11. Experimental circumstances



Fig. 12. A sample input image of the caregiver

\section{Result of learning}

1) Categories of internal state: In results obtained for changes in internal state space, Fig. 14 shows facial expressions corresponding to representational space of the internal state. The robot's internal state is expressed lying along the $(p, a)$ planes. Nodes of the internal state are colored differently, with $(1,0),(1,1),(-1,1)$, and $(-1,0)$ corresponding to blue, green, yellow and white. The caregiver's faces are all associated with distinct behavioral categories in the internal state, distributed similarly to Russell's two-dimensional emotional model.

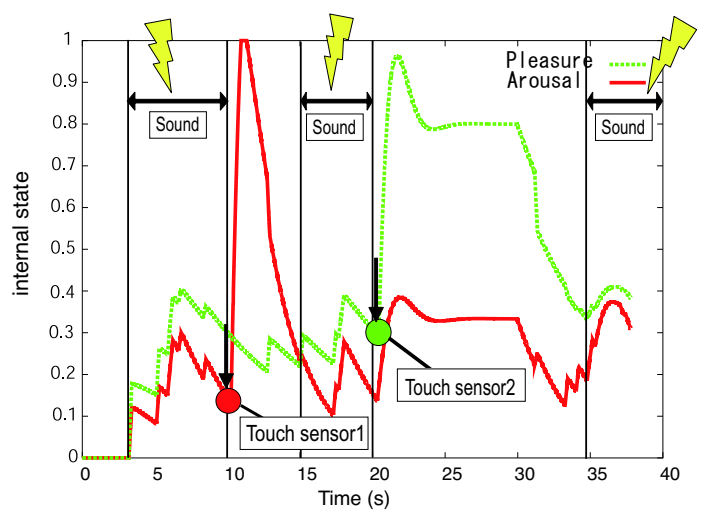

Fig. 13. Transition of internal state



Fig. 14. Facial expressions associated with internal state

2) Categories of facial expressions: Fig. 15 shows internal states associated with the face SOM. The internal state associated with each node of the SOM is changed by the background color that corresponds to those shown in Fig. 14. Since color

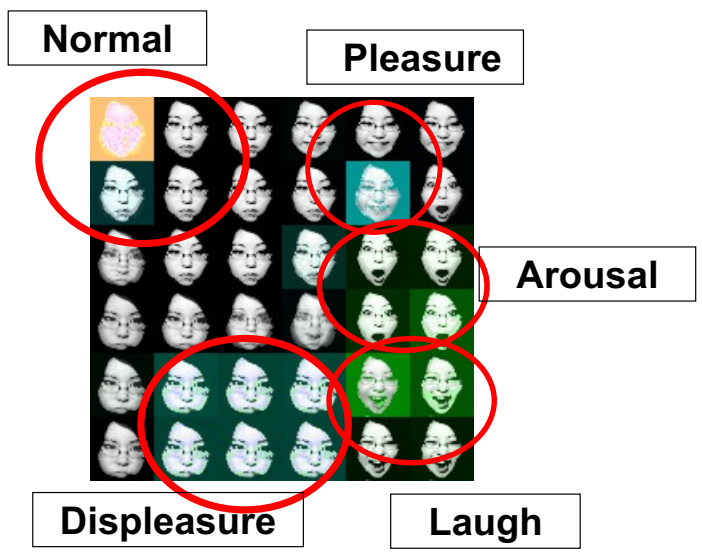

Fig. 15. Associated internal states on face SOM

density is indicative of the strength of the connection, uncolored nodes indicate that the SOM node is not associated with any internal states, or that the weight assigned for Hebbian learning is dispersed.

\section{Interaction after learning}

After learning, the robot assesses the internal human state using the facial expression, and changes its facial expression based on the internal state evoked within the human being. Fig. 16 shows changes in internal state variables and facial expressions during communication with the caregiver after learning over time. The robot changes its internal state based on the caregiver's facial expression and reacts to it as expected. For the first 12 seconds, the experimenter presented the robot with facial features characteristic of laughter. This was reflected as a transition in the robot's internal state to pleasure and arousal, and ultimately, as rough on its face. For the next 13 seconds, the experimenter expressed displeasure. This was reflected by a gradual decrease in pleasure $(p)$, and ultimately 


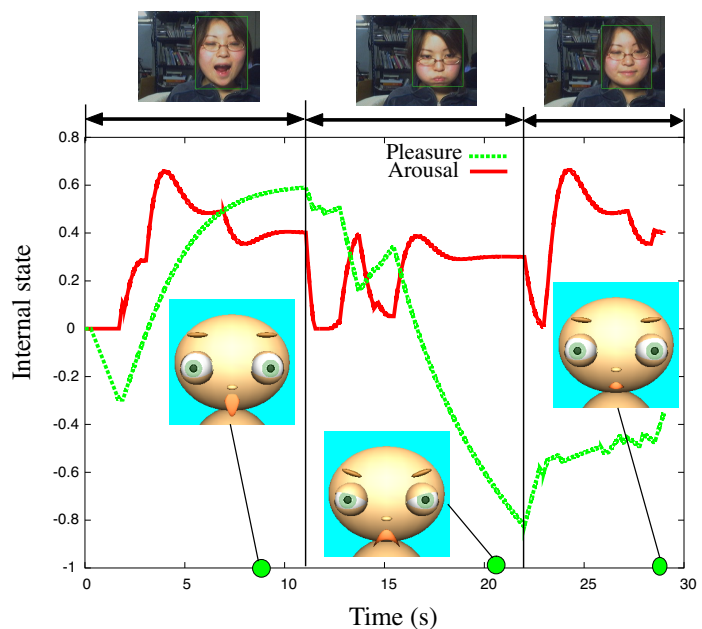

Fig. 16. Sympathetic communication after learning

as a look of displeasure on the robot's face. The experimenter then expressed pleasure for 10 seconds, reflected as no change in arousal $(a)$ and an increase in pleasure $(p)$, resulting in an expression of slight surprise on the robot's face. These results demonstrate that the robot was able to learn how to modify its internal state in response to human facial expressions and to synchronize human facial expressions.

\section{Discussion}

1) Distinction for facial expression: We developed a robot that was able to classify human facial expressions and learning using the proposed model. The robot could not, however, distinguish between ambiguous or weak facial expressions because facial expressions are recognized by differences in the luminance of low-resolution images. The robot was thus only able to distinguish between five different facial expressions, with instances in which only the eyes are distinctive classified inaccurately. The number of facial-expression categories is thus small when internal states are expressed in sequential space. These prevent the robot from learning the facial expressions corresponding to midpoints in the internal state, such as when the eyes are the most important feature, as in anger. These facial expressions were not strongly associated with the internal state (Fig. 14). The algorithm used for distinguishing between different facial expressions must thus be improved to improve the robot's response to subtle changes in facial expression.

Apart from technical issues in facial expression recognition, it is interesting why caregivers show exaggerated expressions (artificial expressions) in early communication. In intuitive parenting, caregivers show exaggerated rather than facial natural expressions. This exaggerated attitude toward an infant is general for caregivers, such as motherese or caregiver's gestures. Infants appear to acquire natural communication skills by generalizing these exaggerated expressions, and caregivers appear to unconsciously aid their infants in learning. These roles of exaggerated expressions in learning are currently under exploration in developmental psychology.
2) Dynamics of internal state: Internal state dynamics are important in our research. The communication between robots and users is unnatural when compared to that observed between human beings because the dynamics associated with a robot's internal state differ from those related to human internal states when exposed to an external stimulus. We studied internal state dynamics based on the interest degree model of an infant, in which the robot's internal state increases when an agent perceives an external stimulus and gradually decreases thereafter. In biological organisms, the underlying system for affection involves numerous regions of the brain and a variety of chemical substances whose the interactions are not understood well enough to enable them to be applied to robotics. The internal state we have proposed is considered representative of real complex systems because it is, to some extent, based on the observations of human behavior. Cooperation between researchers in robotics and those involved in the cognitive or brain sciences may result in the development of models that more accurately reflect the cognitive aspects required to improve the response of robots to human behavior. To this end, we are currently developing a more realistic affect model.

3) Correspondence to neuroscience: What part of the brain is responsible for sympathy? Using fMRI, Singer et al. [16] show brain activity associated with understanding by the subject, of another person's pain. In this experiment, brain activity was observed when a subject was administered a painful stimulus through a electrode on the back of the hand and when the subject was administered the stimulus associated with a simultaneous view of the subject's loved one (Fig. 17).

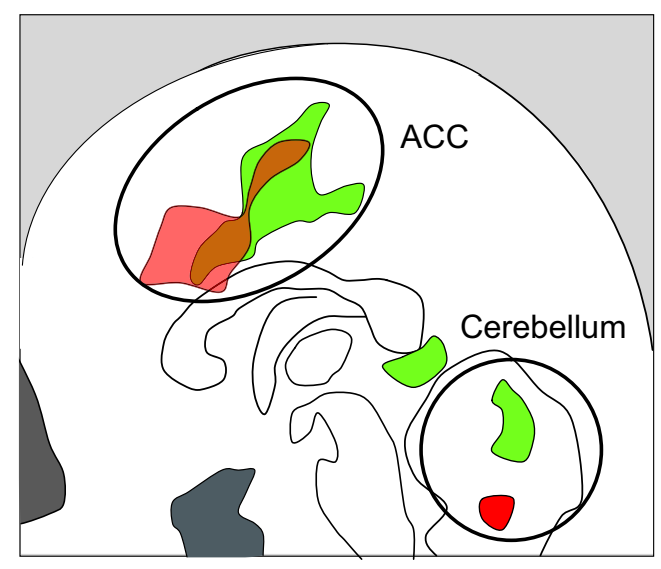

Fig. 17. Activity of brain in response to a pain stimulus [Tania Singer et al. 2004]

Areas in green are activated when subjects were administered a painful stimulus, and areas in red are activated when subjects observed painful stimuli being administered to others. The anterior cingulate cortex (ACC) and the cerebellum are activated in both cases, in addition to somato sensory cortex, but the sensation of pain associated with observing the pain of someone else differs from that of experiencing pain oneself in the same region. These results suggest that the area associated with feeling the pain of others and oneself are the ACC 
and/or the cerebellum, and that human beings, although able to identify with pain felt by others, experience the two differently.

In our proposed model, a robot learned the connection between an experienced internal state and the resultant facial expression of a caregiver in communication similar to that of intuitive parenting. This may correspond to learning the connection between the ACC, through which human beings feel pain, and the temporo parietal cortex (TPJ), which is thought to be responsible for recognizing facial expressions. After learning, the brain region for pain is activated just by observing others' facial expression. In our proposed model, the robot cannot differentiate between its own pain and that of others. This contradicts the findings of Tania Singer et al. [16] who found that parts of the brain in the same area are activated when subjects experience their own pain to self and that of others. While it has not been shown how the notions of "me" and "you" develop, it may be that the following developmental process involves the ACC: (1) The pain area is stimulated first using self-sensor input. (2) Another sensor input of a different modality (e.g. visual information from a caregiver) is partly projected on to the same area. (3) Intuitive parenting strengthens the connection between the subject's feeling based on the pain sensation of self and the feeling toward that of others based on visual information. (4) Sensor input from different modalities suppress each other, finally forming a sympathetic region. In this naive hypothesis, our proposed model may correspond to the third process above.

\section{CONCLUSIONS}

We have discussed intuitive parenting in early communication between infants and their caregivers in the context of modeling the learning process of a robot attempting to associate its internal state with the facial expression of a user. In such learning, the robot's internal state is influenced by a variety of stimuli in response to which the robot adopts specific facial expressions, that are mimicked or exaggerated by the user. This exchange of visual information enables the robot to associate its own internal state with the user's facial expression. The robot then evoke a particular internal state in the user based on a human facial expression and expresses its internal state in response to the effects of the user. Our next goal is a more advanced communication model based on the "sympathetic" concept we have proposed .

\section{REFERENCES}

[1] G. Gergely and J. S. Watson, "Early socio-emotional development: Contingency perception adn the social-biofeedback model," in Early Social Cognition: Understanding Others in the First Months of Life, P. Rochat, Ed. Mahwah, NJ: Lawrence Erlbaum, 1999, pp. 101-136.

[2] A. Mehrabian, Implicit communication of emotions and attitudes. Wadsworth, 1981.

[3] M. H. Johnson and J. Morton, "Biology and cognitive development," Blackwell, 1991.

[4] D. Rosenstein and H. Oster, Differential facial responses to four basic tastes in newborns. Blackwell Publishing, Dec. 1988, vol. 59, pp. $1555-1568$.

[5] D. Matsui, T. Minato, K. F. MacDorman, and H. Ishiguro, "Generating natural motion in an android by mapping human motion," Proc. of IEEE/RSJ International Conference on Intelligent Robots and Systems, pp. 1089-1096, 2005.
[6] T. Hashimoto, M. Sennda, and H. Kobayashi, "Realization of realistic and rich facial expressions by face robot," 2004 1st IEEE Technical Exhibition Based Conference on Robotics and Automation, pp. 37-38, Nov. 2004.

[7] C. Breazeal, D. Buchsbaum, J. Gray, D. Gatenby, and B. Blumberg, "Learning from and about others: Towards using imitation to botstrap the social understanding of others by robots," Artificial Life, vol. 11, pp. 31-62, 2005.

[8] T. Kobayashi, Y. Ogawa, K. Kato, and K. Yamamoto, "Learning system of human facial expression for a family robot," in Proceeding of the Sixth International Conference on Automatic Face and Gesture Recognition, 2004, pp. 481-486.

[9] H. Papousek and M. Papousek, "Intuitive parenting: a dialectic counterpart to the infant's precocity in integrative capacities," Handbook of Infant Development, pp. 669-720, 1987.

[10] P. Rochat, "The infant's world," Artificial Life, 2001.

[11] M. Asada, K. F. MacDorman, H. Ishiguro, and Y. Kuniyoshi, "Cognitive developmental robotics as a new paradigm for the design of humanoid robots," in Proceeding of the 1st IEEE/RSJ International Conference on Humanoid Robots, 2000, p. CDROM.

[12] J. A. Russell, "A circumplex model of affect," Journal of Personality and Social Psychology, vol. 39, pp. 1161-1178, 1980.

[13] H. Yamada, "Visual information for categorizing facial expression of emotion," Japan Psychology Review, vol. 35, pp. 172-181, 1993.

[14] K. Lorenz, Studies in animal and human behabior. London: Methuen, 1970-1971.

[15] T. Kohonen, "Self-organizing maps," Springer-Verlag Verlin Heidelverg, 1995.

[16] T. Singer, B. Seymour, J. O’Doherty, H. Kaube, R. J. Dolan, and C. D. Frith, "Empathy for pain involves the affective but not sensory components of pain," Science, vol. 303, no. 20, pp. 1157-1162, February 2004. 